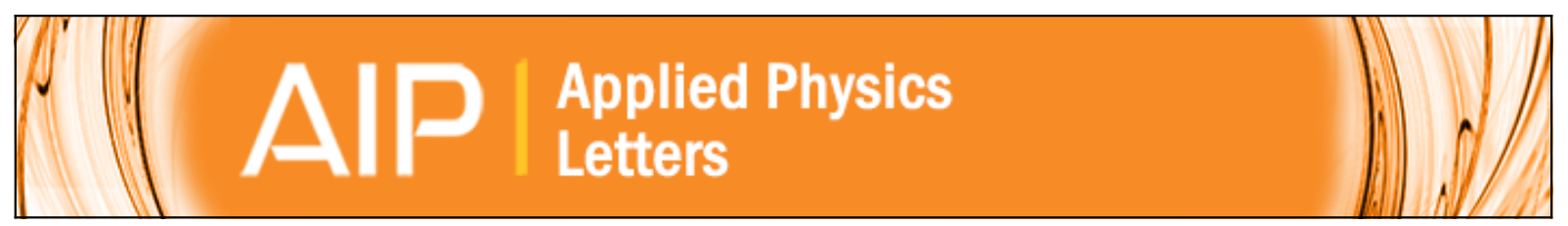

\title{
Electroluminescence from spatially confined exciton polaritons in a textured
}

\section{microcavity}

K. Winkler, C. Schneider, J. Fischer, A. Rahimi-Iman, M. Amthor, A. Forchel, S. Reitzenstein, S. Höfling, and M. Kamp

Citation: Applied Physics Letters 102, 041101 (2013); doi: 10.1063/1.4777564

View online: http://dx.doi.org/10.1063/1.4777564

View Table of Contents: http://scitation.aip.org/content/aip/journal/apl/102/4?ver=pdfcov

Published by the AIP Publishing

$\stackrel{A}{A} \mathbb{P} P$ Re-register for Table of Content Alerts

Create a profile.

Sign up today! 


\title{
Electroluminescence from spatially confined exciton polaritons in a textured microcavity
}

\author{
K. Winkler, C. Schneider, J. Fischer, A. Rahimi-Iman, M. Amthor, A. Forchel, \\ S. Reitzenstein, ${ }^{a}{ }^{2}$ S. Höfling, and M. Kamp \\ Technische Physik, Physikalisches Institut and Wilhelm Conrad Röntgen-Research Center for Complex \\ Material Systems, Universität Würzburg, Am Hubland, D-97074 Würzburg, Germany
}

(Received 11 September 2012; accepted 19 October 2012; published online 28 January 2013)

\begin{abstract}
We report on the formation of spatially confined exciton-polaritons under electrical injection in a textured microcavity. The trapping of polaritons in the diode sample is achieved through a locally elongated GaAs microcavity with a quality factor exceeding 6000 . The polaritonic resonances of traps with diameters of $10 \mu \mathrm{m}$ and $2 \mu \mathrm{m}$ are studied by angular-resolved electroluminescence spectroscopy, revealing their hybrid light-matter nature. (c) 2013 American Institute of Physics. [http://dx.doi.org/10.1063/1.4777564]
\end{abstract}

Strong coupling between quantum well (QW) excitons and photons in a semiconductor microcavity resonator leads to the formation of exciton polaritons. ${ }^{1,2}$ This results in two eigenstates of the coupled system, namely the upper polariton (UP) and lower polariton (LP) branch, ${ }^{3}$ that are split in energy. Due to their bosonic character, the polaritons can undergo a dynamical condensation above a critical particle density resulting in a macroscopically occupied single particle energetic ground state. ${ }^{4,5}$ This condensation is possible at elevated temperatures because of the light effective mass of polaritons, in comparison to excitons or atoms. Since the polaritonic ground state can acquire a high degree of spatial $^{4,6}$ and temporal ${ }^{7-9}$ coherence, such coupled light-matter systems are promising candidates for an attractive kind of laser device, namely the polariton laser. ${ }^{2}$

Trapping polaritons in real space is crucial for the observation of long range coherence phenomena, since long range order over infinite distances cannot exist in a twodimensional system. ${ }^{10}$ While vertical trapping is already achieved by the epitaxial microcavity structure, different approaches have been undertaken to laterally confine polaritons. They involve either trapping the photonic ${ }^{11-13}$ or the excitonic ${ }^{5}$ part of the polariton.

One important aspect for a real device application is the formation of polaritons by means of electrical injection instead of the commonly used optical excitation. This has been demonstrated for quasi-2D polaritons. ${ }^{14-16}$ The unambiguous demonstration of a polariton condensate under electrical pumping has so far been elusive; however, it can be expected that a carefully designed trapping potential in a polariton light emitting diode would be highly beneficial to demonstrate this important milestone. ${ }^{17}$ It has also been predicted that spatially tightly confined polaritons of narrow emission linewidth can emit single photons on demand ${ }^{18}$ or can be exploited in more complex schemes of scalable quantum information processing and quantum cryptography. ${ }^{19}$ This further explains the high interest in advanced and flexible trapping schemes of microcavity exciton polaritons.

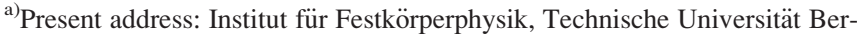
lin, Hardenbergstrasse 36, 10623 Berlin, Germany.
}

Here, we report polariton emission from an electrically driven microcavity comprising a trap that confined the photonic part of the polariton in all three dimensions. The trap design of our structure is based on the confinement of the photonic component through a locally elongated cavity. In this approach, the quantum wells in the microcavity are not damaged by etching through the active region, and detrimental surface effects are hence suppressed. This is in stark contrast to common approaches to confine polaritons in micropillar-cavities, where broadening of the QW emission caused by undesired surface recombination of carriers at etched surfaces is usually significant.

The investigated sample is schematically shown in Fig. 1(a) and was grown by molecular beam epitaxy (MBE). The intrinsic one- $\lambda$ thick GaAs cavity is surrounded by two doped GaAs/AlAs distributed Bragg reflector (DBR) mirrors grown on p-doped GaAs substrate. In the center of the cavity, a $15 \mathrm{~nm}$ thick $\mathrm{Ga}_{0.88} \mathrm{In}_{0.12}$ As quantum well is embedded as the active medium. The lower/upper DBR consists of 27/23 mirror pairs and is $\mathrm{p} / \mathrm{n}$-doped by carbon/silicon to facilitate current injection. To reduce doping related optical absorption in the DBRs, the doping density is reduced from $n=3 * 10^{18} 1 / \mathrm{cm}^{3}$ in the outer layers of the DBR to $n=1 * 10^{18} 1 / \mathrm{cm}^{3}$ near the central cavity layer (indicated in Fig. 1(a) by black to grey scale). The cavity layer and the DBRs are designed for a low temperature resonance wavelength of $\lambda=894 \mathrm{~nm}$ at the center of the wafer. Due to the growth-related decrease in thickness towards the edge of the wafer, the cavity resonance can, however, be tuned down to a minimum wavelength of $\lambda=857 \mathrm{~nm}$.

Quantization of polaritons is achieved by processing the sample in a manner that the photonic component of polaritons is confined in all three spatial dimensions. For this purpose, the cavity length is locally elongated in form of circular mesas with diameters of 0.5 to $10 \mu \mathrm{m}$ and a height of $5 \mathrm{~nm}$. This results in a spatially energy-wise lowered cavity resonance and therefore in a 3D-confinement of the photonic polariton component via photonic traps. ${ }^{11}$

To realize such traps, we extended the process strategy outlined before. ${ }^{20}$ After growth of the bottom DBR and the full intrinsic one- $\lambda$ cavity, the sample was transferred out of 


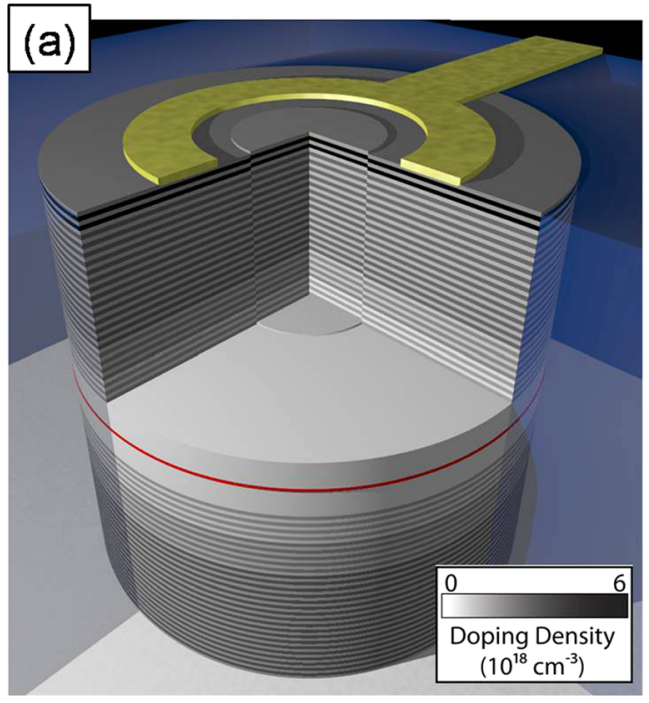

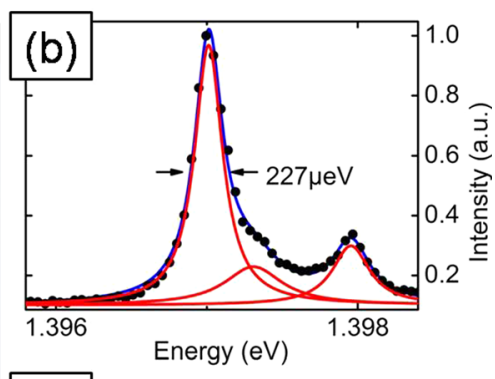

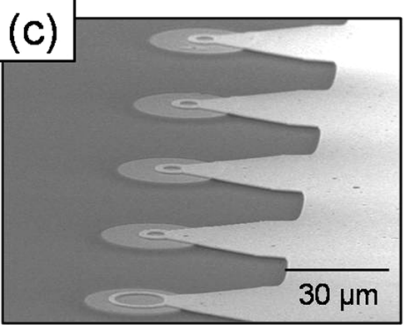

FIG. 1. (a) Schematic sketch of the sample design. The doping levels are indicated by white (intrinsic) to black (high doping) color coding. The QW is drawn in red color. (b) Fitted ground mode photoluminescence emission spectrum of a $10 \mu \mathrm{m}$ trap. The linewidth of $227 \mu \mathrm{eV}$ corresponds to a quality factor of 6150. (c) Scanning electron microscope (SEM) image of a sample processed for electrical injection. The image shows pillars of $30 \mu \mathrm{m}$ diameter with gold contact rings around the centered traps. the MBE machine. Next, we performed position dependent reflection measurements at room temperature to estimate the layer thicknesses in the bottom DBR and the cavity by comparing the reflection spectrum with transfer-matrix calculations. If the determined cavity resonance matches the excitonic emission energy within the radial tuning range, the full 3 in. wafer is processed in order to define the optical traps prior to overgrowth with the top DBR. First, the full wafer is patterned with alignment marks and large mesa structures via optical lithography and wet etching. ${ }^{21}$ These alignment marks serve as reference points for the subsequent electron beam lithography (EBL) step, in which circular mesas with diameters between $500 \mathrm{~nm}$ and $10 \mu \mathrm{m}$ are defined. After developing the $\mathrm{EBL}$ resist and the evaporation of an $\mathrm{Al}$ mask, a lift-off procedure was performed, resulting in $\mathrm{Al}$ disks of the given diameters. The $\mathrm{Al}$ disk pattern was then transferred into the semiconductor by wet etching (using a highly diluted GaAs selective etchant), resulting in mesas of $\sim 5 \mathrm{~nm}$ height, and the $\mathrm{Al}$ circles were removed subsequently. Prior to overgrowth of these mesas, activated hydrogen cleaning of the surface was carried out in the load lock chamber of the MBE to remove surface oxides and contaminations. ${ }^{21}$
The quality factor (Q-factor) of the structure was extracted experimentally at a large cavity-exciton detuning $\mathrm{E}_{\mathrm{c}}-\mathrm{E}_{\mathrm{x}}<-8.02 \mathrm{meV}$ by means of micro-photoluminescence $(\mu \mathrm{PL})$ spectroscopy. Fig. 1(b)) depicts a spectrum of the highly photonic fundamental mode of a $10 \mu \mathrm{m}$ diameter trap, from which we can extract a Q-factor of $\sim 6150$.

The final process of electrical contacting includes dryetching of larger pillars with a diameter of $30 \mu \mathrm{m}$ around the traps and planarization of the sample with isolating benzocyclobutene. The positioning of the large pillars relative to the buried mesas for polariton trapping is facilitated by the alignment marks initially patterned into the structure. The contacting is carried out by gold ring structures on top of the pillar structures (shown in Fig. 1(c)) and a gold backcontact. A row of five different trap sizes can be electrically driven in parallel by means of a common contact pad.

The advantages of this trap fabrication technique are the possibility to study emission from two dimensional (planar) polaritons at the same spot as emission from the traps, and that the traps can be two-dimensionally arranged in arbitrary configurations. This technological route is therefore also very promising to define tailored two-dimensionally
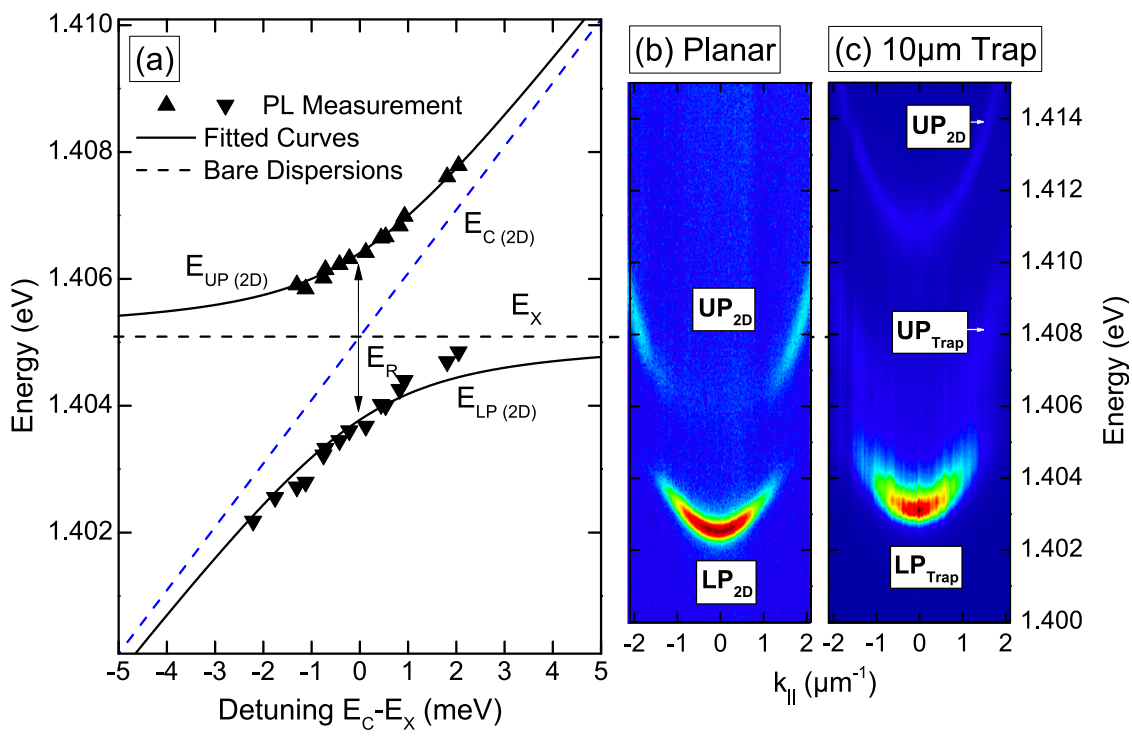

FIG. 2. Characterization of the planar polaritons in means of 2D photoluminescence measurements. (a) Anticrossing of upper and lower polariton extracted from position resolved investigations. (b) Momentum resolved dispersion of the planar polariton at a negative detuning of $-1.77 \mathrm{meV}$. (c) Dispersion of the trapped polariton recorded at a detuning of $-0.9 \mathrm{meV}$. 

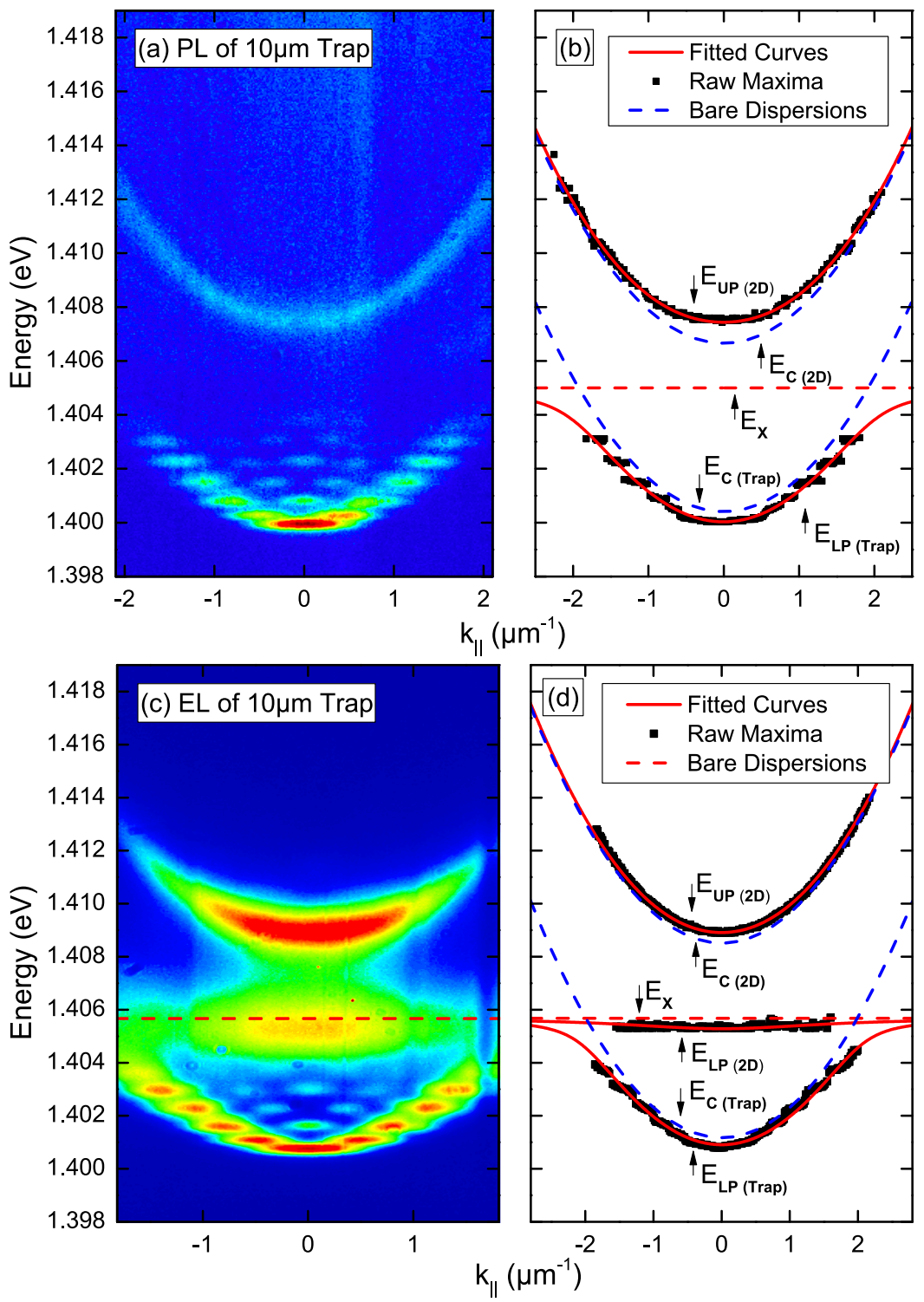

FIG. 3. Emission pattern from a trap with diameter of $10 \mu \mathrm{m}$. Comparison of (a) photoluminescence and (c) electroluminescence measurements. The false-color images show quantized trap states as well as continuous branches from planar background. The raw maxima are fitted in (b) (PL) and (d) (EL) by the theoretical polariton dispersions. patterned potential landscapes ${ }^{19,22,23}$ where the electrical contact could be utilized for local Stark-tuning of the exciton resonance and therewith serve as a tool for fast external manipulation.

To map out the tuning behavior and the Rabi-splitting of the polariton branches, we performed $\mu \mathrm{PL}$ measurements in a momentum-space spectroscopy setup on traps and planar regions by continuous-wave excitation with a continuous wave $(\mathrm{CW})$-diode laser emitting at a wavelength of $658 \mathrm{~nm}$. The sample is mounted in a helium-flow cryostat with electrical feedthroughs, at temperatures of 5-8 K. This method allows the investigation of momentum-space dependent energy dispersions and, thus, makes it possible to identify lower and upper polariton branches and distinguish between trap emission and planar emission.

Fig. 2(a) shows the typical anticrossing behavior of upper and lower planar polaritons in the region around zero detuning between cavity mode $\left(\mathrm{E}_{\mathrm{c}}\right)$ and exciton $\left(\mathrm{E}_{\mathrm{x}}\right)$ energy at $\mathrm{k}_{\|}=0$ obtained from momentum resolved photoluminescence measurements as depicted exemplarily in Fig. 2(b) for a negative detuning of $-1.77 \mathrm{meV}$. In the investigated energy range, the exciton energy can be treated as constant since it shifts significantly less $(\sim 0.2 \mathrm{meV})$ than the photon resonance $(\sim 5.5 \mathrm{meV})$. Fitting the experimental data (solid lines in Fig. 2(a)) yields a Rabi-splitting energy $\mathrm{E}_{\mathrm{R}}=2.63 \pm 0.08 \mathrm{meV}$.

The energy dispersion of a trap device with a diameter of $10 \mu \mathrm{m}$ at a moderate red detuning of $-0.9 \mathrm{meV}$ recorded under optical excitation is exemplary shown in Fig. 2(c). Due to the large effective mass of the polaritons in this regime, the direct influence of the trapping potential on the dispersion is not immediately visible. However, some mode quantization already evolves close to the fundamental resonance, consistent with reports on similar devices. ${ }^{11,20}$ The visibility of the quantized modes is strongly pronounced in the red detuning regime $\left(\mathrm{E}_{\mathrm{c}}-\mathrm{E}_{\mathrm{x}} \sim-4.6 \mathrm{meV}\right)$ as depicted in Fig. 3(a): The photon confinement leads to energy modes quantized in energy and momentum space, ${ }^{24}$ in contrast to the continuous dispersion of the planar polariton in Fig. 2(b). Because of the comparatively large trap size of $10 \mu \mathrm{m}$, the momentum-quantized eigenenergies of the ground mode still resemble a two-dimensional dispersion. The luminescence of the highly excitonic planar LP is outshined by the bright emission of the photonic UP and the emission from the 


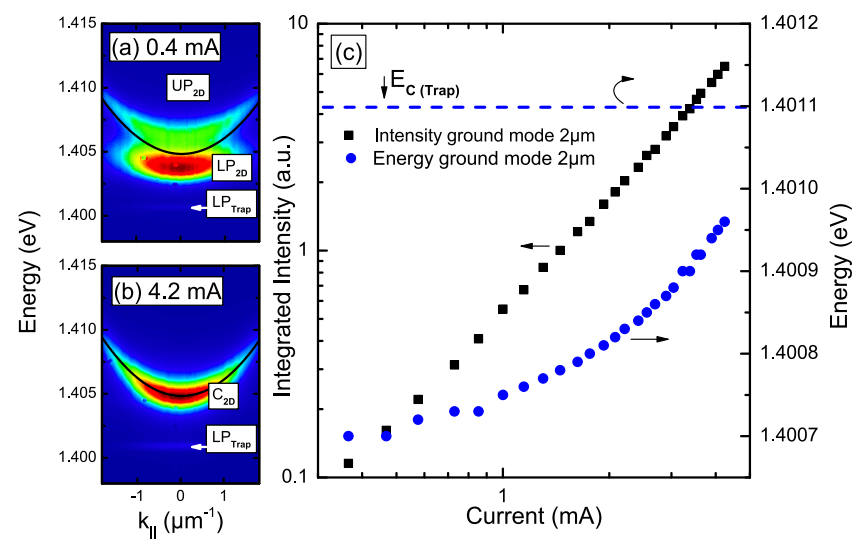

FIG. 4. Power-dependent EL of a $2 \mu \mathrm{m}$ trap. At low injection currents, the Rabi splitting is obvious in the planar background emission (a), while the dispersion becomes photon-like (indicated by black line) at high pump rates (b). (c) Power-dependent integrated intensity and energy shift of the ground mode.

trapped LP. This is a consequence of the optical excitation scheme with a spot of $\sim 4 \mu \mathrm{m}$ diameter, which predominantly generates excitons in the region of the polariton trap. The maxima of this dispersion are fitted together with the planar background and the results are shown in Fig. 3(b). From this evaluation, we can extract a trapping potential of photonic fundamental mode of $\Delta \mathrm{E}_{\mathrm{C}}=6 \mathrm{meV}$ in agreement with transfer-matrix computations on the planar devices and a Rabi-splitting of $2.76 \mathrm{meV}$, which is comparable to the planar structure.

Fig. 3(c) depicts the energy momentum dispersion from an electrically contacted device at a similar detuning $\left(\mathrm{E}_{\mathrm{c}}-\mathrm{E}_{\mathrm{x}} \sim-4.5 \mathrm{meV}\right)$, acquired under electrical injection. The diode structure was forward biased at a current of $0.68 \mathrm{~mA}$ in $\mathrm{CW}$ mode. The emission pattern, quality factors, and the evaluation results of the electroluminescence (EL) spectra (Fig. 3(d)) are comparable to the one obtained from PL data. In EL, the ground-mode shows a linewidth of $\sim 330 \mu \mathrm{eV}$, which is in full agreement with the polariton linewidth of $\sim 315 \mu \mathrm{eV}$ extracted from $\mu \mathrm{PL}$ measurements in Fig. 3(a). However, the Rabi splitting is slightly reduced to $\sim 2.3 \mathrm{meV}$ which is attributed to enhanced exciton screening effects caused by free carriers in the electrical injection scheme at the selected excitation conditions.

We further investigated the behavior of the system under variation of the injection current for a $2 \mu \mathrm{m}$ trap (Fig. 4) at a negative detuning of $-4.2 \mathrm{meV}$. For this trap size, only the fundamental polaritonic trap mode is observable because the energy of the photonic ground mode is increased by approximately $2 \mathrm{meV}$ (compared to the $10 \mu \mathrm{m}$ diameter traps), and the energy splitting of the photonic resonances is predicted to be $\sim 5 \mathrm{meV}$. For low injection currents (Fig. 4(a)), the strong coupling regime directly manifests itself in the planar background emission signal, the dispersion of which strongly deviates from a weakly coupled photonic parabola dispersion (black line, Fig. 4(a)). Furthermore, the planar polariton is split into the upper and lower branch, which are both visible at these excitation conditions. The Rabi-splitting reduces with injection current due to exciton screening and a successive reduction of the exciton oscillator strength, ${ }^{25}$ when the system enters the weak coupling regime. This is obvious from the high-excitation power spectrum recorded at an injection current of $4.2 \mathrm{~mA}$ in Fig. 4(b), where only one dominant branch can be observed in the planar emission, which can be approximated by the purely photonic parabolic dispersion.

The transition from strong coupling to weak coupling is accompanied by the blueshift of the trapped polaritonic fundamental mode (Fig. 4(c)). A continuous blueshift of this mode towards the weakly coupled photonic trap energy position (dashed blue line) is observable in the selected pumping range. In order to assess the nonlinear properties of the system, the integrated intensity emitted from the polariton trap is plotted as a function of the injection current in double logarithmic style. The input-output characteristics of the device can be approximated by a super-linear power dependence with $\mathrm{I} \sim \mathrm{J}^{1.6}$, where $\mathrm{I}$ is the integrated intensity and $\mathrm{J}$ denotes the injection current through the device. This distinct power law behavior is observed over almost two orders of magnitude of intensity, from the clearly polaritonic regime into the transition regime from strong to weak coupling. We attribute the deviation from the expected linear input-output behavior to an enhancement of polariton-phonon scattering towards the energetic polariton groundstate: The loss of translational invariance caused by the spatial confinement results in an enhanced overlap of adjacent polariton wavefunctions in momentum space, and hence the stringent conditions on phonon $\mathrm{k}$-vectors involved in the scattering process are lifted. ${ }^{17}$

In conclusion, we demonstrated emission from trapped exciton-polaritons under electrical injection. The trapconfinement is realized by a locally elongated, circular cavity, which can be conveniently contacted to facilitate electrical pumping and manipulation. The polaritonic character of the emission is confirmed both in the emission from the trap states, as well as the planar background emission under optical and electrical pumping. Enhanced scattering towards the groundstate in such a low-dimensional system is identified by its nonlinear input-output characteristics, pointing towards the possibility to observe polariton condensation under electrical injection in a sample with a larger number of quantum wells.

This work has been supported by the State of Bavaria. Technical assistance by T. Steinl and M. Emmerling during sample preparation is gratefully acknowledged. We thank V. D. Kulakovskii for fruitful discussions.

${ }^{1}$ C. Weisbuch, M. Nishioka, A. Ishikawa, and Y. Arakawa, Phys. Rev. Lett. 69, 3314-3317 (1992).

${ }^{2}$ A. Kavokin, J. J. Baumberg, G. Malpuech, and F. P. Laussy, Microcavities (Oxford University Press, 2007).

${ }^{3}$ T. C. H. Liew, I. A. Shelykh, and G. Malpuech, Physica E 43, 1543-1568 (2011).

${ }^{4}$ J. Kasprzak, M. Richard, S. Kundermann, A. Baas, P. Jeambrun, J. M. J. Keeling, F. M. Marchetti, M. H. Szymańska, R. André, J. L. Staehli, V. Savona, P. B. Littlewood, B. Deveaud, and L. S. Dang, Nature 443, 409414 (2006).

${ }^{5}$ R. Balili, V. Hartwell, D. Snoke, L. Pfeiffer, and K. West, Science 316, 1007-1010 (2007).

${ }^{6}$ H. Deng, D. Press, S. Götzinger, G. Solomon, R. Hey, K. Ploog, and Y. Yamamoto, Phys. Rev. Lett. 97, 146402 (2006).

${ }^{7}$ H. Deng, G. Weihs, C. Santori, J. Bloch, and Y. Yamamoto, Science 298, 199-202 (2002).

${ }^{8}$ M. Assmann, J.-S. Tempel, F. Veit, M. Bayer, A. Rahimi-Iman, A. Löffler, S. Höfling, S. Reitzenstein, L. Worschech, and A. Forchel, Proc. Natl. Acad. Sci. U.S.A. 108, 1804-1809 (2011). 
${ }^{9}$ J. Kasprzak, M. Richard, A. Baas, B. Deveaud, R. André, J.-P. Poizat, and L. Dang, Phys. Rev. Lett. 100, 067402 (2008).

${ }^{10} \mathrm{P}$. Hohenberg, Phys. Rev. 158, 383-386 (1967).

${ }^{11}$ O. El Daï, A. Baas, T. Guillet, J.-P. Brantut, R. I. Kaitouni, J. L. Staehli, F. Morier-Genoud, and B. Deveaud, Appl. Phys. Lett. 88, 061105 (2006).

${ }^{12}$ N. Y. Kim, C.-W. Lai, S. Utsunomiya, G. Roumpos, M. Fraser, H. Deng, T. Byrnes, P. Recher, N. Kumada, T. Fujisawa, and Y. Yamamoto, Phys. Status Solidi B 245, 1076-1080 (2008).

${ }^{13}$ T. Gutbrod, M. Bayer, A. Forchel, and J. P. Reithmaier, Phys. Rev. B 57, 9950-9956 (1998).

${ }^{14}$ D. Bajoni, E. Semenova, A. Lemaître, S. Bouchoule, E. Wertz, P. Senellart, and J. Bloch, Phys. Rev. B 77, 113303 (2008).

${ }^{15}$ S. I. Tsintzos, P. G. Savvidis, G. Deligeorgis, Z. Hatzopoulos, and N. T. Pelekanos, Appl. Phys. Lett. 94, 071109 (2009).

${ }^{16}$ A. A. Khalifa, A. P. D. Love, D. N. Krizhanovskii, M. Skolnick, and J. Roberts, Appl. Phys. Lett. 92, 061107 (2008).

${ }^{17}$ D. Bajoni, J. Phys. D 45, 313001 (2012).
${ }^{18}$ A. Verger, C. Ciuti, and I. Carusotto, Phys. Rev. B 73, 193306 (2006).

${ }^{19}$ N. Na and Y. Yamamoto, New J. Phys. 12, 123001 (2010).

${ }^{20}$ A. Rahimi-Iman, C. Schneider, J. Fischer, S. Holzinger, M. Amthor, S. Höfling, S. Reitzenstein, L. Worschech, M. Kamp, and A. Forchel, Phys. Rev. B 84, 165325 (2011).

${ }^{21}$ C. Schneider, T. Heindel, A. Huggenberger, T. A. Niederstrasser, S. Reitzenstein, A. Forchel, S. Höfling, and M. Kamp, Appl. Phys. Lett. 100, 091108 (2012).

${ }^{22}$ N. Y. Kim, K. Kusudo, C. Wu, N. Masumoto, A. Löffler, S. Höfling, N. Kumada, L. Worschech, A. Forchel, and Y. Yamamoto, Nat. Phys. 7, 681686 (2011).

${ }^{23}$ I. Carusotto, D. Gerace, H. Tureci, S. De Liberato, C. Ciuti, and A. Imamoglu, Phys. Rev. Lett. 103, 033601 (2009).

${ }^{24}$ P. Lugan, D. Sarchi, and V. Savona, Phys. Status Solidi C 3, 2428-2431 (2006).

${ }^{25}$ A. L. Bradley, J. P. Doran, J. Hegarty, R. P. Stanley, U. Oesterle, R. Houdré, and M. Ilegems, J. Lumin. 85, 261-270 (2000). 